\title{
Electrochemical Comparison of the Interaction of 5-Nitrouracil with Single-or Double-Stranded DNA at mercury and glassy carbon electrodes
}

\author{
Mohamed Sayed Ibrahim, Hossieny Sameh Mohamed Ibrahim, Moustafa Mohamed Kamal, Yassin \\ Mohamed Temerk \\ msayed217@yahoo.co.uk Gsm: 00201227690833 Chrmistry Department,faculty Of Science, Assiut \\ University, Assiut, Egypt
}

\begin{abstract}
The interaction of the 5-Nitrouracil (5NU), with ss-, and ds-DNA was investigated electrochemically in absence and presence of copper ions by using cyclic voltammetry (CV) and differential pulse stripping voltammetry (DPSV) at hanging mercury drop electrode (HMDE) and glassy carbon electrode (GCE) surfaces. It was found that, in absence of copper ions, the addition of ss- or ds-DNA to a buffered solution of $5 \mathrm{NU}$ results in a decrease on the $5 \mathrm{NU}$ redox peak current with a remarkable change in the peak potential (ca. $150 \mathrm{mV}$ vs. $\mathrm{Ag} / \mathrm{AgCl}$ ) at both electrodes. This means that, an interaction of 5NU molecules with both ss- and ds-DNA was observed. The results also demonstrate that a distinguish between ss-, and ds-DNA can be achieved in presence of copper ion through their interaction with 5NU. The binding constants of 5NU with ds-DNA at HMDE and GCE were determined through the changes on the 5NU redox peak currents (at HMDE, 1.45×105 and at GCE, 2.65x105). The calibration plot for the DNA determination was obtained through the corresponding decreases on the DPSV peak current of 5NU to different additions of DNA (ss- or ds-DNA) concentration levels at the optimum conditions.
\end{abstract}

Key words: Voltammetry, 5-nitrouracil, interactions, DNA

\section{Introduction}

The bioactivity of 5-substituted uracils, a class of the substituted pyrimidines, induces exceptional interest in their biochemistry and pharmacology[1], and they are the most interesting and studied uracils. 5-substituted uracils are potential substitutes for thymine in the DNA, and are used to increase the sensitivity of the DNA to ionizing radiation[2]. The most prominent representatives are: 5-fluorouracil $[3,4]$ and 5-bromouracil [5,6]. Apart from the halogenmodified nucleobases several aromatic nitro compounds also show promise as radiosensitizers for overcoming the radioprotection afforded some tumor cells by their lack of oxygen, 'hypoxia' [7]. Moreover, many derivatives of 5-nitrouracil have shown the following effects: antibacterial activity [8], antitumor activity on leukemia cells [9] inhibitory effect on macrophage [10], prime interest to the non-linear optical community [11] and relevance to the biological and pharmaceutical sciences [12]. In addition, the 5nitrouracil has been known to inhibit thymidine phosphorylase [13]. The complexing ability of these ligands with some essential bioelements in serum ( $\mathrm{Fe}(\mathrm{III}), \mathrm{Cu}$ (II) etc.), as well as with $\mathrm{Al}(\mathrm{III})$ and $\mathrm{Mg}(\mathrm{II})$ ions in antacids is of special interest. DNA is quite often the major cellular target for studies with smaller molecules of biological importance such as carcinogens, steroids and several classes of drugs [14-16], particularly for the designing of new DNA-targeted drugs and the experience of these in vitro. Drug-DNA interactions have been studied by a variety of analytical techniques with their relative advantages and disadvantages such as luminescence [17], fluorescence [18], UV-vis spectroscopy [19], and voltammetric methods [20]. Among these electrochemical methods are widely used in drug-DNA binding studies due to their advantages like high sensitivity, efficient selectivity, cost affectivity, more reliability, extensive versatility and fast detection ability. So, in the recent years, growing interest has arisen in electrochemical investigations of interaction between nucleic-acid-binding molecules and DNA using especially mercury or carbon electrodes [21-24]. Furthermore, the voltammetric methods were used to probe the interaction (electrostatic or intercalative) of a number of metal chelates with calf thymus DNA [25]. Continuing our studies on the interaction of small 
molecules with DNA [26-29], the present paper is concerned with the voltammetric studies of the

\section{Materials and Method}

\section{$2.1 \quad$ Chemicals and reagents}

Single-stranded (ss) or double-stranded (ds) calf thymus deoxyribonucleic acid (DNA) and 5nitrouracil (Fig. 1) were obtained from Sigma and were used without further purification. Stock solutions of DNA were prepared by dissolving an appropriate amount of the DNA in $\mathrm{H} 2 \mathrm{O}$ and stored at $4 \circ \mathrm{oC}$. DNA concentration (per nucleotide phosphate, NP) was determined interaction of 5-nitrouracil with calf thymus ss- or ds-DNA in absence and presence of $\mathrm{Cu}(\mathrm{II})$.

spectrophotometrically assuming $\varepsilon 260=6600 \mathrm{M}$ $1 \mathrm{~cm}-1$. Stock solution of the $5 \mathrm{NU}$ was prepared by dissolving an appropriate amount of the $5 \mathrm{NU}$ in water. The supporting electrolyte was phosphate buffer prepared in the usual way by adding appropriate amounts of $1.0 \mathrm{M}$ orthophosphoric acid to $0.25 \mathrm{M}$ sodium phosphate. All chemicals were reagent grade (Merck, Darmstadt). Twice-distilled water was used to prepare the aqueous solutions. The $\mathrm{pH}$ was measured using pH-meter Model M64 (ORION) with accuracy to \pm 0.05 .

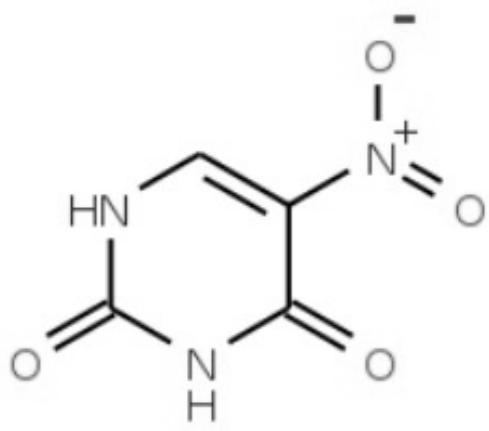

Figure 1.

\section{$2.2 \quad$ Instrumentation}

A PAR Model 264A polarographic analyzer/ stripping voltammetry coupled with a PAR Model 303A HMDE and X-Y recorder Model RE0151 were employed for differential pulse stripping voltammetric (DPSV) and cyclic voltammetric (CV) measurements. The area of the HMDE was $1.2 \times 10-2 \mathrm{~cm} 2$. A PAR glassy carbon electrode (GCE) for Model 303A was also used as the working electrode. $\mathrm{An} \mathrm{Ag} / \mathrm{AgCl}$ saturated $\mathrm{KCl}$ reference electrode and a $\mathrm{Pt}$ wire auxiliary electrode were used. All voltammetric measurements were carried out at the ambient temperature of the laboratory (23-25 oC), according to our previous work [27].

\section{Results and Discussion}

\subsection{The interaction of $5 N U$ with ds-DNA at HMDE}

The interaction of $5 \mathrm{NU}$ with ds-DNA was investigated by $\mathrm{CV}$ and DPSV at the HMDE surface. By investigating the effect of $\mathrm{pH}$ value on the reaction of 5NU with ds-DNA, it was found that in the $\mathrm{pH}$ range from 3.0 to 12.0 , the addition of ds-DNA to 5NU solution and subsequent scanning over the potential range produced no new peaks, but a decrease in reduction peak current with a big shift of the peak potential to a negative direction was observed (Fig.2). As reported previously, the shifted of the peak potential of $\mathrm{CV}$ waves to more positive or negative values, indicating that the action of $5 \mathrm{NU}$ with ds-DNA may be intercalation [30]. Strong interaction are observed at $\mathrm{pH} 3.0$ and 10.0 phosphate buffer compared to media with other $\mathrm{pH}$ values. The latter behaviour reflects that the 
protonated and anionic species of $5 \mathrm{NU}$ are strongly interacted with ds-DNA.

According to these observations, it seems that the decreases of peak currents of $5 \mathrm{NU}$ after an addition of excess ds-DNA are caused by the intercalation of $5 \mathrm{NU}$ to the bulky, slowly diffusing ds-DNA, which results in a considerable decrease in the apparent diffusion coefficient. We suppose this intercalation masks the electroactive site of $5 \mathrm{NU}$ and in a way reduces the equilibrium concentration of 5NU. This behaviour confirmed by the dependence of the $5 \mathrm{NU}$ process at the HMDE on the scan rate $(v)$, in the absence and presence of an excess ds-DNA. The peak currents

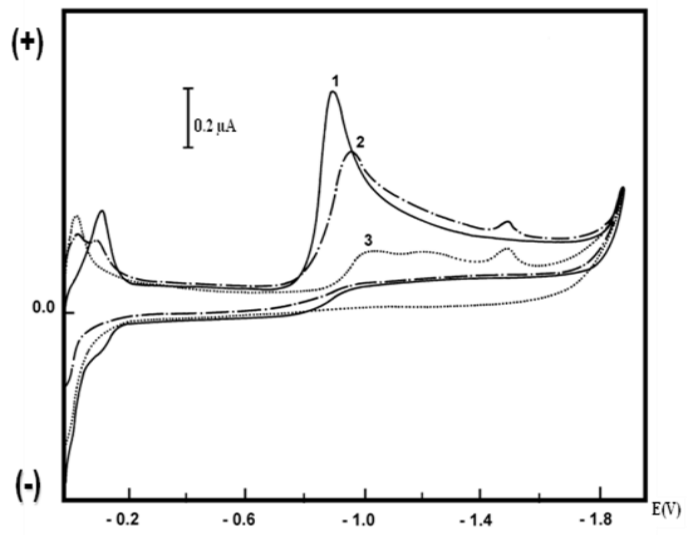

Figure 2.

\subsection{The interaction of $5 N U$ with ds-DNA at \\ GCE}

The interaction of 5NU with ds-DNA was also investigated by CV and DPSV at GCE. Typical differential pulse voltammetric behaviours of $5 \mathrm{NU}$ in the absence, and presence, of ds-DNA at the GCE are shown in Fig. 4. By investigating the effect of $\mathrm{pH}$ value on the reaction of $5 \mathrm{NU}$ with ds-DNA at GCE, it was found that in the $\mathrm{pH}$ range from 3.0 to 12.0 , the addition of ds-DNA to $5 \mathrm{NU}$ solution and subsequent scanning produced no new peaks, but caused the peak current diminish considerably with a large shift of the peak potential to a negative direction. Strong interaction are observed in the acidic media of $\mathrm{pH}$ 3.0 phosphate buffer compared to media with other $\mathrm{pH}$ values. The latter behaviour reflects that the protonated species of 5NU are strongly interacted with ds-DNA at GCE. Moreover, there is no significant obstruction of the glassy carbon surface via adsorption of DNA, as opposed to obtained for $5 \mathrm{NU}$ and 5NU-ds-DNA adduct show linear dependence on the square root of the scan rate up to $500 \mathrm{mV} \mathrm{s}-1$, indicating diffusional mass transport of the electroactive species to the HMDE surface at lower scan rate [31]. Furthermore, the slope of the linear of Ipc versus $v 1 / 2$ without ds-DNA was more than that with dsDNA (Fig. 3), suggesting the diffusion coefficient of the free form of $5 \mathrm{NU}$ (Df) was larger than that of the complexed form of $5 \mathrm{NU}$ with ds-DNA (Db) as shown in Table 1.

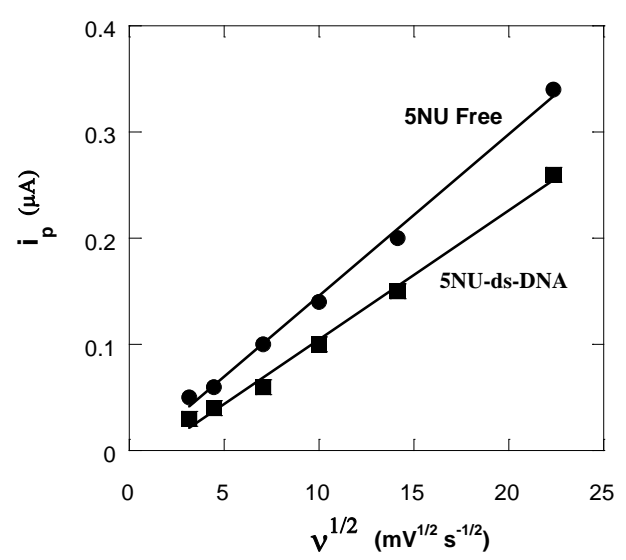

Figure 3.

mercury electrodes, where adsorption of DNA at negative potentials is significant [32]. On one hand, the intercalation of 5NU into a large DNA double helix might increase the steric hindrance to the heterogeneous electron transfer. On the other hand, intercalation might induce some overlap between the large $\pi$-bond of the pyrimidine moiety of $5 \mathrm{NU}$ and the stacked base planes of dsDNA [33]. The probable explanation of these experimental phenomena is that $5 \mathrm{NU}$ intercalated to ds-DNA forms an electroinactive complex with its diffusion coefficient decreasing responsible for the decrease of peak currents [34]. This behaviour confirmed by the dependence of the $5 \mathrm{NU}$ process at the GCE on the scan rate $(v)$, in the absence and presence of an excess ds-DNA. The slope of the linear of Ipc versus $v 1 / 2$ without ds-DNA was more than that with ds-DNA, suggesting the diffusion coefficient of the free form of $5 \mathrm{NU}$ (Df) was larger than that of the complexed form of 5NU with ds-DNA (Db) as shown in Table 1. 
Table 1: Diffusion coefficients of 5NU and 5NU-ds-DNA complex calculated from the results of CV using the slope of the linear of ip versus $v 1 / 2$ in absence and presence of ds-DNA at the HMDE and GCE at $\mathrm{pH} 3.0$.

\begin{tabular}{|c|c|c|c|c|}
\hline Electrode & \multicolumn{2}{|c|}{$\begin{array}{c}\text { Slope } \\
\left(\mathrm{nA} / \mathrm{mV}^{1 / 2} \mathrm{~s}^{-1 / 2}\right)\end{array}$} & $\begin{array}{c}\mathrm{D}_{\mathrm{f}} \\
\left(\mathrm{cm}^{2} \mathrm{~s}^{-1}\right)\end{array}$ & $\begin{array}{c}\mathrm{D}_{\mathrm{b}} \\
\left(\mathrm{cm}^{2} \mathrm{~s}^{-1}\right)\end{array}$ \\
\hline Species & $5 \mathrm{NU}$ & $5 \mathrm{NU}-\mathrm{ds}-\mathrm{DNA}$ & $5 \mathrm{NU}$ & $5 \mathrm{NU}-\mathrm{ds}-\mathrm{DNA}$ \\
\hline HMDE & 15.2 & 12.1 & $1.87 \times 10^{-7}$ & $1.18 \times 10^{-7}$ \\
\hline GCE & 52.4 & 25.6 & $2.22 \times 10^{-6}$ & $5.30 \times 10^{-7}$ \\
\hline
\end{tabular}

\subsection{Calculation of the Binding constant (K)}

Current titrations were performed by keeping the constant concentration of the $5 \mathrm{NU}$ while varying the concentrations of ds-DNA at HMDE or GCE using DPSV at pH 3 (Fig's. 4 and 5). The interaction of $5 \mathrm{NU}$ with ds-DNA can be described using the following equation:

$$
5 \mathrm{NU}+\mathrm{ds}-\mathrm{DNA} \leftrightarrow
$$

5NU-ds-DNA

An equation for amperometric titration can be deduced according to references $[9,35]$. The

controlled electron transfer and the complex of $5 \mathrm{NU}$ with ds-DNA (in nucleotide phosphate) is a $1: 1$ association complex, then the plot of $\log$ $1 /$ dds-DNA] vs. log IH-G/IG-IH-G becomes linear with the intercept of $\log \mathrm{K}$. Based on the variations in the currents at the HMDE and GCE (Fig's. 4 and 5), the binding constants of these complexes were evaluated according to equation (2) and the results are listed in Table 2. The results indicate the large binding constant for 5NU-ds-DNA adduct at GCE than that obtained at HMDE.

$$
\log \frac{1}{[\mathrm{ds}-\mathrm{DNA}]}=\log \mathrm{K}+\log \frac{\mathrm{I}_{\mathrm{H}-\mathrm{G}}}{\mathrm{I}_{\mathrm{G}}-\mathrm{I}_{\mathrm{H}-\mathrm{G}}}
$$

current titration equation was described as follows Where $\mathrm{K}$ is the apparent binding constant, IG and IH-G are the peak current of the free guest $(\mathrm{G}$; here free $5 \mathrm{NU}$ ) and the host-guest complex (H-G; $5 \mathrm{NU}$ intercalated into ds-DNA), respectively. Under the assumption of reversible, diffusion-

The change in Gibbs free energy, $\Delta \mathrm{G}^{\circ}$, is an important parameter which reflects the stability of the formed adduct. $\Delta \mathrm{G}^{\circ}$ can be derived from equation (3):

$$
\Delta \mathrm{G}^{\circ}=-\mathrm{RT} \ln \mathrm{K}
$$

Where the $\mathrm{R}$ is the universal constant $(8.31 \mathrm{~J} \mathrm{~K}-1$ mol-1), $\mathrm{T}$ the absolute temperature, and $\mathrm{K}$ the binding constant. The $\Delta \mathrm{G}^{\circ}$ values for the binding of $5 \mathrm{NU}$ with the ds-DNA were calculated at both HMDE and GCE (Table 2). These values are negative indicating that the binding process is favourable and spontaneous. 


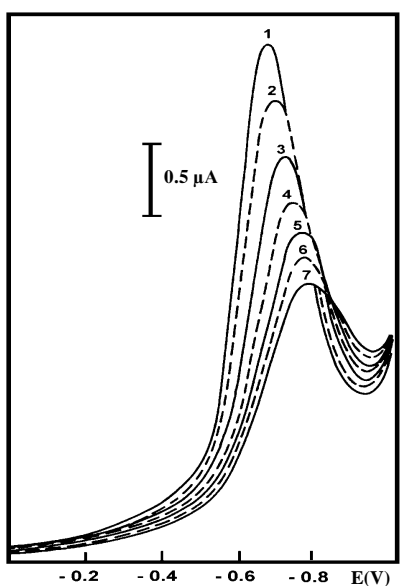

Figure 4.

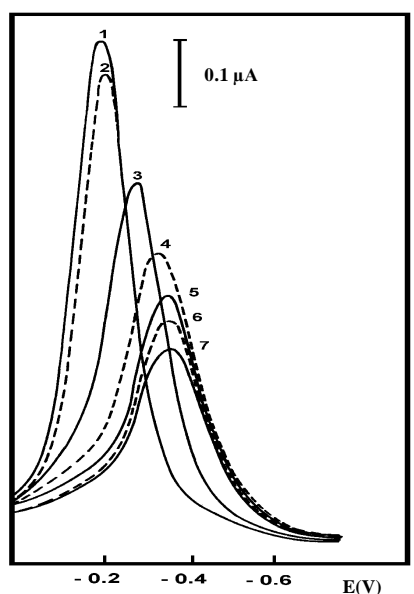

Figure 5.

Table 2: Binding constants of 5NU-ds-DNA complex calculated from the results of DPSV at the HMDE and GCE at pH 3.0.

\begin{tabular}{|c|c|c|}
\hline Electrode & $\mathrm{K}\left(\mathrm{M}^{-1}\right)$ & $-\Delta \mathrm{G}\left(\mathrm{kJ} \mathrm{mol}^{-1}\right)$ \\
\hline HMDE & $1.45 \times 10^{5}$ & 29.46 \\
\hline GCE & $2.65 \times 10^{5}$ & 30.95 \\
\hline
\end{tabular}

\subsection{Effect of $\mathrm{Cu}(\mathrm{II})$ on the interaction of $5 \mathrm{NU}$ with ss- or ds-DNA at $\mathrm{HMDE}$}

The differential pulse stripping voltammograms of 5NU, 5NU-DNA, and 5NU-DNA-Cu at $\mathrm{pH} 3.0$ are shown in Fig's 6A and 6B. As increasing the ss- or ds-DNA concentration at constant $5 \mathrm{NU}$ concentration, a decrease in reduction peak current with a big shift of the peak potential to a negative direction was observed. This means that, an interaction of 5NU molecules with both ss- and ds-DNA was observed. On the other hand, the addition of $\mathrm{Cu}$ (II) ions decrease the interaction between 5NU and ss-DNA and a positive potential shift was observed (Fig. 6A), indicating that the action of $5 \mathrm{NU}$ with ss-DNA may be electrostatic interaction by which $5 \mathrm{NU}$ molecules bind electrostatically to the negatively charged deoxyribose-phosphate backbone of DNA. However, the interaction of 5NU with ds-DNA approximately unaffected by the addition of $\mathrm{Cu}$ (II) ions as show in Fig. 6B. This behaviour can confirm the suggested interaction mode of $5 \mathrm{NU}$ molecules with ds-DNA by intercalation (by which $5 \mathrm{NU}$ intercalated into the double helix of DNA). We suppose this intercalation masks $5 \mathrm{NU}$ molecules and in a way reduces the interaction between $\mathrm{Cu}$ (II) and 5NU. From these characteristics, it is apparent that intercalative and groove bindings are related with the grooves in the DNA double helix while the electrostatic binding can take place out of the groove or on the surface of the DNA molecule. Thus distinguish between the interaction mode of ss-, and ds-DNA can be achieved in presence of copper ions through their interaction with $5 \mathrm{NU}$.

\subsection{Linear Range of DNA Determination}

The decrease in peak current of 5NU resulted from the addition of ss-DNA or ds-DNA into the $5 \mathrm{NU}$ solution can be employed to determine the concentration of ss-DNA or ds-DNA. The peak current of the DPSV peak of 5NU was used as the detection signal. Under the optimum experimental conditions of Fig's. 4 and 5, the decreases in the DPSV peak current were linearly related to ssDNA or ds-DNA concentration in the range of 0 $-30 \mu \mathrm{M}$ when the $5 \mathrm{NU}$ concentrations were fixed at 2.0x10-5 M, with detection limits of $1.23 \times 10-6$ $\mathrm{M}$ for ds-DNA and 2.66x10-7 $\mathrm{M}$ for ss-DNA. The procedure to handle a sample takes only 15-20 min. The proposed method is simple, sensitive and rapid and hence can be applied to the determination of many kinds of DNA. 


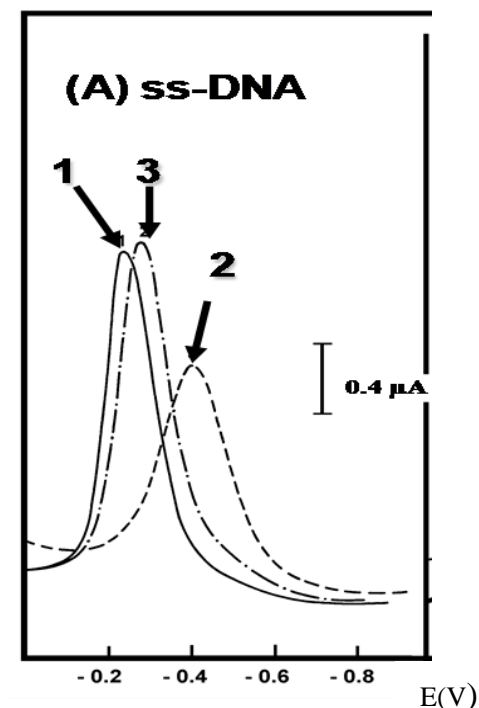

Figure 6A.

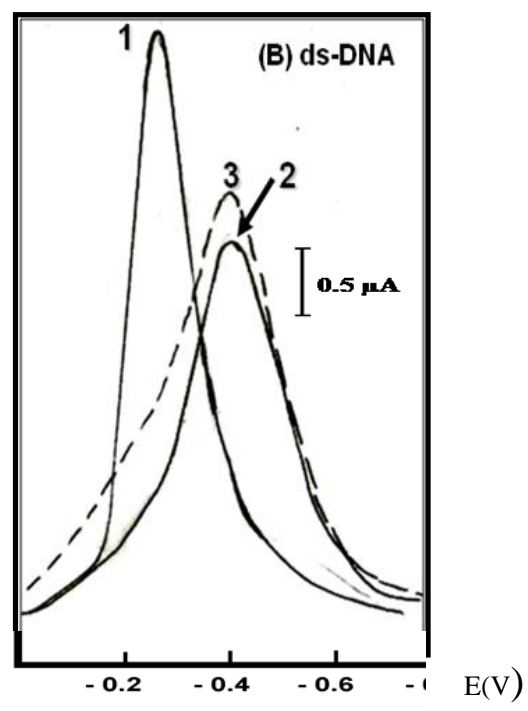

Figure 6B.

\subsection{The nature of $5 \mathrm{NU}$ interacting with DNA}

This work has shown experimental evidence of interaction of 5NU with DNA at HMDE and GCE, and may contribute to the understanding of the mechanism of action of this drug with ss- and ds-DNA in absence and presence of copper ions. In comparison to the HMDE, 5NU displayed high affinity for ds-DNA at the GCE. The large binding constant $(\mathrm{K})$ observed for $5 \mathrm{NU}$ at GCE in compared to that at HMDE (Table 2) is attributed to a mode of binding that involves a strong stacking interaction between the 5NU chromophore and the base pairs of ds-DNA, i.e. the 5NU chromophore is in close proximity to the ds-DNA bases at the GCE surface. The results presented here show also that, the distinguish between the interaction mode of ss-, and ds-DNA can be achieved in presence of copper ions through their interaction with 5NU. The voltammetric changes unequivocally suggest that $5 \mathrm{NU}$ is intercalated into the base-stacking domain of the ds-DNA double helix, however, the interaction mode of $5 \mathrm{NU}$ molecules with ss-DNA is mainly by electrostatic (by which $5 \mathrm{NU}$ bind electrostatically to the negatively charged deoxyribose-phosphate backbone of DNA). It has been reported that the intercalation of DNA with small molecules commonly has stronger strength than electrostatic action [36], thus, the 5NU molecules should have stronger affinity with dsDNA than ss-DNA. This results are consistent with that reported previously by Ortiz M. et al. [37] for the single-stranded DNA interaction with methylene blue using spectroscopic technique. In summary, the interaction is correlated to the planarity, hydrophobicity and electrostatic component of $5 \mathrm{NU}$ and the main effect on the interaction is the intercalation (by hydrophobic interaction) of $5 \mathrm{NU}$ to DNA. These investigation are of potential importance in understanding the mechanism of interaction and recognition of the drugs in the living body.

\section{References}

[1] Katzung BA. Basic and Clinical Pharmacology, 6th ed. (Appleton \& Lang, Norwalk,CT; 1995.

[2] Lawrence TS, Davis MA, Maybaum J, Stetson PL, Ensminger WD. The Effect of Single

versus Double-Strand Substitution on Halogenated Pyrimidine-Induced Radiosensitization and DNA Strand Breakage in Human Tumor Cells. Radiat Res 1990; 123: 1928.

[3] Jain KS, Chitre TS, Miniyar PB, Kathiaravan MK, Bendre VS, Veer VS, et al. Biological and medicinal significance of pyrimidines. J Curr Sci 2006; 90(6):793-803.

[4] Longley DB, Harkin DP, Johnston PG. 5Fluorouracil: mechanisms of action and clinical strategies. Nat Rev Can 2003; 3:330-8.

[5] Buchsbaum DJ, Khazaeli MB, Davis MA, Lawrence TS. Sensitization of radiolabeled monoclonal antibody therapy using bromodeoxyuridine.Cancer 1994; 73: 999-1005.

[6] Chelladurai M, Lobocki CA, Sultani M, Hanna Y, Drelichman A, Pieper DR, et al. Bromodeoxyuridine improves the cytotoxic effect 
of cisplatin: a comparison with 5-fluorouracil. Cancer Chemother Pharmacol 1997; 40(6):463-8. [7] Wardman P, Clarke ED, Flockhart IR, Wallace RG. The rationale for the development of improved hypoxic cell radiosensitizers. Brit J Cancer 1978 (Suppl. III) 1-5.

[8] Lee BH, Shin JH, Lim MK, Jang TS, Park JS, $\mathrm{Kim} \mathrm{KH}$, et al. Bull. Partition property of 5nitrothiopyrimidine Nucleoside. Bull Korean Chem Soc 1979; 18(7): 734-6.

[9] Jiménez BM, Kranz P, Lee CS, Gero AM, O'Sullivan WJP. Inhibition of uridin phosphorylase from Giardia lamblia by pyrimidine analogs. Biochem Pharmacol 1989; 38(21): 3785-9.

[10] Copik A, Suwinski J, Walczak K, Bronikowska J, Czuba Z, Krool W. Synthesis of 1-(2-hydroxy-3-methoxypropyl)uracils and their activity against L1210 and macrophage raw 264.7 cells. Nucleosides Nucleotides Nucleic Acids 2002; (21): 377-383.

[11] Puccetti G, Perigaud A, Badan J, Ledoux I, Zyss J. 5-Nitrouracil: a transparent and efficient nonlinear organic crystal. J Opt Soc Am B 1993; 10 (4): 733- 744.

[12] Smiley JA, Angelot JM, Cannon RC, Marshall EM, Asch DK. Radioactivity-based and spectrophotometric assays for isoorotate decarboxylase: identification of the thymidine salvage pathway in lower eukaryotes. Anal Biochem 1999; 266(1): 85-92.

[13] Coats E, Glave WR, Hansch C. Structureactivity relations in thymidine phosphorylase inhibitors. A correlation using substituent constants and regression analysis. Med J Chem 1970; 13(5): 913-9.

[14] Gills JJ, Lopoiccolo J, Denni PA. Nelfinavir, a new anti-cancer drug with pleiotropic effects and many paths to autophagy. Autophagy 2008; 4(1):107-9.

[15] Hurley LH. DNA and its associated processes as targets for cancer therapy. Nat Rev Cancer 2002; 2(3): 188-200.

[16] Shahabadi N, Moghadam NH. Determining the mode of interaction of calf thymus DNA with the drug sumatriptan using voltammetric and spectroscopic techniques. Spectrochimica Acta Part A: Molecular and Biomolecular Spectroscopy 2012; 99:18-22.

[17] Arkin MR, Stemp EDA, Turro C, Turro NJ, Barton JK. Luminescence Quenching in Supramolecular Systems: A Comparison of DNAand SDS Micelle-Mediated Photo-induced Electron Transfer between Metal Complexes. J Am Chem Soc 1996; 118: 2267-74.

[18] Zhong W, Yu JH, Liang Y. Chlorobenzylidine-herring sperm DNA interaction: binding mode and thermodynamic studies. Spectrochim. Acta Part A 2003; 59(6): 1281-8.

[19] Shah A, Zaheer M, Qureshi R, Akhter Z, Nazar MF. Voltammetric and spectroscopic investigations of 4-nitrophenylferrocene interacting with DNA. Spectrochimica Acta Part A 2010; 75:1082-7.

[20] Aslanoglu M, Oge N. Voltammetric, UV Absorption and Viscometric Studies of the Interaction of Norepinephrine with DNA. Turk J Chem 2005; 29: 477-85.

[21] Mirmomtaz E, Ensafi AA. Voltammetric determination of trace quantities of 6-thioguanine based on the interaction with DNA at a mercury electrode. Electrochimica Acta 2009; 54: 4353-8. [22] Ensafi AA, Rezaei B, Amini M, HeydariBafrooei E. A Novel Sensitive DNA-Biosensor For Detection of a Carcinogen, Sudan II, Using Electrochemically Treated Pencil Graphite Electrode by Voltammetric Methods. Talanta 2012; 88: 244-251.

[23] Bian CL, Zeng QX, Yang LJ, Xiong HY, Zhang XH, Wang SF. Voltammetric studies of the interaction of rutin with DNA and its analytical applications on the MWNTs-COOH/Fe3O4 modified electrode. Sensors and Actuators B 2011; 156(2): 615-620.

[24] Wang Y, Ni Y, Kokot S. Voltammetric behavior of complexation of salbutamol with calf thymus DNA and its analytical application. Analytical Biochemistry 2011; 419(2): 76-80.

[25] Carter MT, Rodriguez M, Bard AJ. Voltammetric studies of the interaction of metal chelates with DNA. 2. Tris-chelated complexes of cobalt(III) and iron(II) with 1,10-phenanthroline and 2,2'-bipyridine. J Am Chem Soc 1989; 111(24): 8901-11.

[26] Ibrahim MS. Voltammetric studies of the interaction of nogalamycin antitumor drug with DNA. Anal Chim Acta 2001; 443:63-72.

[27] Ibrahim MS, Kamal MM, Temerk YM. Comparison of the voltammetric studies at mercury and glassy carbon electrodes for the interaction of lumichrome with DNA and analytical applications. Anal Bioanal Chem 2003; 375:1024-30.

[28] Temerk YM, Ibrahim MS, Kotb M. Voltammetric and spectroscopic studies on binding of antitumor Morin, Morin-Cu complex and Morin-beta-cyclodextrin with DNA. Spectrochim Acta A 2009; 71 (5):1830-6.

[29] Temerk YM, Ibrahim MS, Kotb M, Schuhmann W. Interaction of antitumor

flavonoids with dsDNA in the absence and presence of $\mathrm{Cu}(\mathrm{II})$. Anal Bioanal Chem 2013; 405(11):3839-46.

[30] Pang DW, Abruna HD. Micromethod for the investigation of the Interactions between DNA 
and Redox-Active Molecules. Anal Chem 1998; 70:3162-9.

[31] Bard AJ, Faulkner LR. Electrochemical Methods Fundamentals and Applications. Wiley, New York 1980.

[32] Frei YF, Miller IR. Influence of Adsorbed

Positively Charged Polyelectrolytes on Polarographic Currents of Cationic Depolarizers. II. J Phys Chem 1965; 69(9): 3018-23.

[33] Wang ZY, Han R, in: Pharmaceutical Treatment of Tumor (in Chinese), People's Health Press, Beijing, 1987; 72-4.

[34] Zhu Z, Li NQ. Electrochemical studies of 9,10-anthraquinone interacting with hemoglobin and determination of hemoglobin. Mikrochim Acta 1999; 130(4): 301-8.

[35] Feng Q, Li NQ, Jiang YY. Electrochemical studies of porphyrin interacting with DNA and determination of DNA. Anal Chim Acta 1997; 344:97-104.

[36] Schäfer S, Sheldrick WS. Coligand tuning of the DNA binding properties of halfsandwich organometallic intercalators: influence of polypyridyl (pp) and monodentate ligands $(\mathrm{L}=\mathrm{Cl}$, (NH2)2CS, (NMe2)2CS) on the intercalation of ( 75 -pentamethylcyclopentadienyl)iridium (III)dipyridoquinoxaline and-dipyridophenazine complexes. J Organ Chem 2007; 692: 1300-9.

[37] Ortiz M, Fragoso A, Ortiz PJ, O’Sullivan CK. Elucidation of the mechanism of single stranded DNA interaction with methylene blue: A spectroscopic approach. J Photochem Photobio A: Chem 2011; 218: 26-32. 